\title{
A Survey of Truancy at Special School for Children with Intellectual Disability in Japan
}

\author{
Takanobu SAKaI ${ }^{1, *}$, Masayoshi TsugE ${ }^{2}$, \\ Sae Kouchiyama ${ }^{3}$, and Shigeki Sonoyama ${ }^{4, \dagger}$
}

${ }^{1}$ Faculty of Human Sciences, Konan Women's University, Japan

${ }^{2}$ Faculty of Human Sciences, University of Tsukuba, Japan

${ }^{3}$ Graduate School of Comprehensive Human Sciences, University of Tsukuba, Japan

${ }^{4}$ Faculty of Humanities and Education, The University of Shimane, Japan

\begin{abstract}
The number of truant elementary and lower secondary school students in Japan may be as high as 120,000, and measures to manage these students are in place, such as the provision of school counselors. Numerous research papers on truancy have been published; however, little information is available on truant students at schools for special needs education, because few studies have been conducted on truancy and methods for supporting truant students at such schools. In this study, we conducted a questionnaire survey on truant students and invited the participation of all schools for special needs education (intellectual disabilities: ID) (which comprise the majority of all schools for special needs education in Japan). The percentage of schools with truant students tends to be smaller for schools for special needs education (ID) than for ordinary elementary and lower secondary schools. However, the percentage of truant students is higher at schools for special needs education (ID). The study shows that the causes of truancy at schools for special needs education (ID) are different from those at ordinary elementary and lower secondary schools; moreover, schools for special needs education (ID) typically have insufficient coordinated, in-school support mechanisms for truant students. These results suggest that truancy at schools for special needs education (ID) is different than at ordinary schools, and this paper discusses the need to study the reality of the truancy of individual students and effective methods for providing needed support.
\end{abstract}

Key Words: truancy, school for special needs education, intellectual disability

\section{Introduction}

The Child Welfare Act in Japan was promulgated in 1947, after which child guidance centers were established across the country. Records of counseling for children who disliked school, provided at the child guidance centers in each prefecture, began to appear in 1957 (Takagi, 1983). In 1966, the Ministry of Education, Science and Culture (currently, the Ministry of Education, Culture, Sports, Science and Technology) conducted the School Basic Survey

\footnotetext{
* Corresponding Author

Mailing Address: 6-2-23, Morikita-cho, Higashinada-ku, Kobe-shi, Hyogo 658-0001, Japan

E-mail Address: t.sakai@konan-wu.ac.jp

${ }^{\dagger}$ Formerly Faculty of Human Sciences, University of Tsukuba, Japan

Received January 17, 2018, Accepted February 16, 2019

DOI: $10.6033 /$ specialeducation. 8.1
}

for the first time, wherein it defined students who disliked school for psychological reasons and were therefore absent from school for extended periods (more than 50 days per year), for example, "dislike of school (学校嫌い gakkou-girai).” However, beginning with the 1991 survey, this period was reduced to 30 days, and beginning in 1998, the term "dislike of school" was changed to "truancy (不登校 futoukou)" (Ministry of Education, Culture, Sports, Science and Technology [MEXT], 2017a). In the early years of this annual survey, the number of truant students remained almost flat; however, this number began to increase in 1984, and this increasing trend was especially notable in the case of lower secondary schools. According to the Results of 2016 Survey on Issues Regarding Student Guidance Concerning Student's Behavior Problems and Truancy, conducted by MEXT, the number of truant students in School Year 2017 was 31,151 at elementary schools (truancy 
rate: $0.5 \%$; the truancy rate represents truant students as a percentage of the total number of enrolled students), 103,247 at lower secondary schools (truancy rate: $3.0 \%$ ), and 48,579 at upper secondary schools (truancy rate: 1.5\%) (MEXT, 2017b). In this survey, truant students were defined as those absent from school, continuously or intermittently, for more than 30 days in a school year (excluding students who were absent for financial or medical reasons); this definition of truancy includes students who want to attend school but do not or cannot due to psychological, emotional, physical, and/or social factors. In School Year 1991 (April 1991 to March 1992), when the above definition of truant students was first used, the combined truancy rate for elementary and lower secondary schools was $0.47 \%$. The rate increased to $1.06 \%$, exceeding $1.0 \%$ for the first time, in School Year 1998 and has since remained between 1.11\% and $1.35 \%$, never again dropping below 1\% (MEXT, 2017b). A detailed nationwide survey investigating the reason for truancy, type of school, and region (prefecture) has been conducted on a continual basis at elementary, lower secondary, and upper secondary schools. A system to support truant students has been developed, including the employment of specialists such as school counselors and social workers (MEXT, 2015).

However, a considerably limited number of studies have been conducted on truancy at schools for special needs education. Furthermore, according to a review of the literature, no nationwide surveys focusing on truancy at schools for special needs education have been conducted (Sonoyama, Cho, \& Kuramitsu, 2017). In Japan, the population of young children (aged 0 to 14 years) has been decreasing since 1980, but the numbers of schools for special needs education and students attending these schools have been increasing (MEXT, 2017c). The number of schools for special needs education that provide educational support for students with intellectual disabilities (ID) accounts for $66.9 \%$ of all schools for special needs education, and students with ID account for $90.0 \%$ of all students at schools for special needs education (MEXT, 2015). Einfeld, Ellis, and Emerson (2011) reported that $20 \%$ to $50 \%$ of students with ID had multiple mental disorders, including depression, anxiety-related disorders, other emotional disorders, and/or behavioral disorders. These mental disorders are not necessarily direct causes of truancy; how- ever, we cannot rule out the possibility that they are related to the persistent truancy of students with ID and serve as contributing factors. Based on the results of the School Basic Survey, Sonoyama et al. (2017) reported that the truancy rate at schools for special needs education (ID) had been increasing; they also mentioned that a very limited number of studies had been conducted on truant students at schools for special needs education and stressed the necessity for such studies.

In this study, we conducted a nationwide questionnaire survey of truant students at schools for special needs education (ID) in Japan to produce a summary outline of the number of truant students, truancy rates, circumstances contributing to truancy, and support systems for truant students as well as reveal any other information that could be used to support truant students at schools for special needs education (ID).

\section{Methods}

\section{Schools Surveyed and Period of Survey}

From February 10 to March 15, 2017, we conducted a questionnaire survey and invited the participation of all 855 schools (including 58 branch schools) for special needs education for students with ID in Japan. The respective schools selected respondents.

\section{Questionnaire Survey Method and Questions}

In schools for special needs education, elementary, lower secondary, and upper secondary departments are established according to elementary school, junior high school, and high school; thus, schools that included more than one department were asked to provide responses based on each department. The schools were primarily asked to provide information on the following and then return the questionnaire by mail: (1) number of students by department and grade in School Year 2016 (April 2016 to March 2017); (2) number of truant students by grade in School Year 2016; (3) support systems available at school (including whether a committee was in place, and if so, whether the committee collaborated with specialized, external institutions (e.g., child counseling center, education counseling center, medical clinic) and/or experts); (4) time and duration of truancy for individual students; (5) causes of tru- 
ancy for individual students; (6) circumstances of students after they became truant; and (7) details of support activities found to be effective for individual truant students (the schools were asked to provide this information only if they had students who had been truant during School Year 2016 but had later returned and currently attending school). In a case that the school had more than six truant students, they were asked to report on up to five truant students for the questionnaire topics 4 to 7 to reduce the burden on survey respondents.

\section{Ethical Considerations}

We sent a document to the schools explaining the purpose of the survey and that the questionnaire was voluntary, along with a consent form to be signed by the school principals; the document also asked that the questionnaire and consent form be returned by mail.

\section{Method of Analysis}

Responses were received from 383 schools (response rate: $44.8 \%$ ), and 379 of these responses were considered valid and therefore analyzed. Information related to questionnaire topics 4 to 6 was provided for 835 students. Since schools for special needs include varying combinations of departments, an analysis was performed on a department-level basis. The percentage of schools that had truant students in School Year 2016 and the percentage of truant students at these schools were calculated for each grade.

\section{Results}

Of the 379 schools with valid responses, 292 (86.1\%) had truant students in School Year 2016. Table 1 provides an overview of the results for each department level. The number and percentage of schools with truant students and the total number and percentage of truant students were uniformly highest for the upper secondary department, second highest for the lower secondary department, and lowest for the elementary department level.

Table 2 shows the number of truant students in each grade and the percentage they represent of the total enrollment in their respective departments. Both the number and percentage of truant students for the elementary department increased as students
Table 1 Summary of Truant Students in Schools for Special Needs Education (ID)

\begin{tabular}{cccc}
\hline Department & Elementary & $\begin{array}{c}\text { Lower } \\
\text { secondary }\end{array}$ & $\begin{array}{c}\text { Upper } \\
\text { secondary }\end{array}$ \\
\hline Number (school/student) & $277 / 12,611$ & $272 / 10,249$ & $339 / 25,422$ \\
\hline Schools with truant students & 75 & 99 & 292 \\
& 27.1 & 36.4 & 86.1 \\
\hline \multirow{2}{*}{ Truant students } & 157 & 203 & 967 \\
& 1.2 & 2.0 & 3.8 \\
\hline
\end{tabular}

Note. top: number, bottom: percentage (\%).

Table 2 Truant Students in Each Grade

\begin{tabular}{lccc}
\hline & $\begin{array}{c}\text { Elementary } \\
n=12,611\end{array}$ & $\begin{array}{c}\text { Lower secondary } \\
n=10,249\end{array}$ & $\begin{array}{c}\text { Upper secondary } \\
n=25,422\end{array}$ \\
\hline Grade 1 & 16 & 61 & 309 \\
& 0.1 & 0.6 & 1.2 \\
Grade 2 & 24 & 84 & 362 \\
& 0.2 & 0.8 & 1.4 \\
Grade 3 & 25 & 58 & 296 \\
& 0.2 & 0.6 & - \\
Grade 4 & 29 & - & - \\
& 0.2 & & - \\
Grade 5 & 22 & - & \\
& 0.2 & & \\
Grade 6 & 41 & - & \\
& 0.3 & & \\
\hline
\end{tabular}

Note. top: number of students, bottom: percentage (\%).

advanced to higher grades; however, for the lower secondary department and upper secondary department, these numbers and percentages peaked in the second grade.

Notably, 154 schools (40.6\%), including one school with a committee not focused specifically on truancy and one school with an ad hoc committee, had a committee to address truancy; 237 schools (62.5\%) collaborated with specialized, external institutions, and/or experts; 127 schools (33.5\%) did not have a committee in place, but collaborated with specialized external institutions and/or experts; and 98 schools (25.9\%) neither had a committee in place nor collaborated with either specialized external institutions or experts.

In School Year 2016, for the questionnaire topics 4 to 7 , the number of students, about whom the school had provided a response, was 99 in the elementary 
Table 3 Causes of Truancy at Each Department

\begin{tabular}{|c|c|c|c|c|}
\hline & & $\begin{array}{c}\text { Elementary } \\
\qquad n=99\end{array}$ & $\begin{array}{c}\text { Lower } \\
\text { secondary } \\
n=149\end{array}$ & $\begin{array}{c}\text { Upper } \\
\text { secondary } \\
n=587\end{array}$ \\
\hline \multirow{10}{*}{ School-related causes } & \multirow{2}{*}{ Difficulty in learning } & 4 & 14 & 68 \\
\hline & & 4.0 & 9.4 & 11.6 \\
\hline & \multirow{2}{*}{ Relationships with other students } & 14 & 37 & 153 \\
\hline & & 14.1 & 24.8 & 26.1 \\
\hline & \multirow{2}{*}{ Relationships with teachers } & 10 & 22 & 54 \\
\hline & & 10.1 & 14.8 & 9.2 \\
\hline & \multirow{2}{*}{ Bullying } & 0 & 2 & 11 \\
\hline & & 0.0 & 1.3 & 1.9 \\
\hline & \multirow{2}{*}{ Other school-related difficulties } & 7 & 25 & 113 \\
\hline & & 7.1 & 16.8 & 19.3 \\
\hline \multirow{6}{*}{ Family-related causes } & \multirow{2}{*}{ Parents' difficulty in sending students to school } & 4 & 67 & 243 \\
\hline & & 4.0 & 45.0 & 41.4 \\
\hline & \multirow{2}{*}{ Parents not eager to send students to school } & 36 & 49 & 152 \\
\hline & & 36.4 & 32.9 & 25.9 \\
\hline & \multirow{2}{*}{ Parents do not want their child's teachers } & 11 & 6 & 8 \\
\hline & & 11.1 & 4.0 & 1.4 \\
\hline \multirow{10}{*}{ Student-related causes } & \multirow{2}{*}{ Apathy } & 1 & 21 & 123 \\
\hline & & 1.0 & 14.1 & 21.0 \\
\hline & \multirow{2}{*}{ Stubborn unwillingness to attend school when they are urged to } & 26 & 55 & 154 \\
\hline & & 26.3 & 36.9 & 26.2 \\
\hline & \multirow{2}{*}{ Activities for amusement/delinquency } & 25 & 6 & 31 \\
\hline & & 25.3 & 4.0 & 5.3 \\
\hline & \multirow{2}{*}{ Day-night reversal } & 12 & 51 & 187 \\
\hline & & 12.1 & 34.2 & 31.9 \\
\hline & \multirow{2}{*}{ Social withdrawal } & 0 & 35 & 176 \\
\hline & & 0.0 & 23.5 & 30.0 \\
\hline
\end{tabular}

Note. top: number of students, bottom: percentage (\%).

Multiple answers allowed if there are multiple causes.

department, 149 in the lower secondary department, and 587 in the upper secondary department (835 students in total). Table 3 shows the causes of truancy (multiple answers were allowed) for each department. Of the school-related causes, "Relationships with other students" ranked highest for all departments. The truancy cause "Relationships with teachers" ranked second highest for the elementary department, third highest for the lower secondary department, and fourth highest for the upper secondary department. We observe that school-related truancy causes other than "Relationships with teachers" tended to become more impactful in the higher grades. In particular, "Difficulty in learning" contributed more significantly to truancy among older students and was the third highest school-related reason for truancy in the upper secondary department. "Other school-related difficulties" accounted for a significant number of truancy cases in all departments.

For the family-related causes, "Parents not eager to send students to school" contributed to a rela- 
A Survey of Truancy at Special School for Children with Intellectual Disability in Japan

Table 4 Circumstances of Students after They Became Truant

\begin{tabular}{lccc}
\hline \multicolumn{1}{c}{ Circumstances of students after they became truant } & Elementary & Lower secondary & Upper secondary \\
$n=587$ & $n=149$ & 34 \\
\hline Attend classes continuously & 3 & 6.0 & 5.8 \\
\hline Attend classes in a different room almost every day & 3.0 & 4 & 10 \\
\hline Attend classes intermittently (including attending in a different room) & 2 & 2.7 & 1.7 \\
\hline Unable to attend classes but able to go out & 4.0 & 69 & 254 \\
\hline Withdraw from society and almost always stay at home & 40.4 & 43 & 43.3 \\
\hline \hline
\end{tabular}

Note. top: number of students, bottom: percentage (\%).

Multiple answers allowed if there are multiple causes.

Table 5 Support Activities Effective for Each Department

\begin{tabular}{lccc}
\hline & Elementary & Lower secondary & Upper secondary \\
$n=53$ & $n=14$ & 7 & 32 \\
\hline Home visits by classroom teachers and others & 6 & 50.0 & 60.4 \\
\hline Parents are eager to have their child attend school & 66.7 & 4 & 14 \\
\hline Student interviews (counselling) by classroom teachers and others & 4 & 28.6 & 26.4 \\
\hline Parent interviews (counselling) by classroom teachers and others & 22.2 & 7 & 34 \\
\hline Support from specialized, external institutions and/or experts & 77.8 & 10 & 64.2 \\
\hline
\end{tabular}

Note. top: number of students, bottom: percentage (\%).

Multiple answers allowed if there are multiple effective support activities.

tively high number of truancy cases in all departments, whereas the impact of "Parents do not want their child's teacher to teach them" decreased as children advanced to higher grades. "Parents' difficulty in sending students to school" began to contribute to a large number of truancy cases in the lower secondary department and continued to do so in the upper sec- ondary department.

For the student-related causes, "Stubborn unwillingness to attend school when they are urged to" ranked highly for all departments, whereas the percentage of total truancy cases that "Day-night reversal" contributed to increased dramatically from the elementary department to the lower secondary 
department. "Social withdrawal" did not contribute to any truancy cases in the elementary department, but its contribution to truancy increased dramatically in the lower secondary department, with a further dramatic increase in the upper secondary department. "Activities for amusement/delinquency" ranked highly among truancy causes in the elementary department, but its percentage was not particularly high in the lower and upper secondary departments.

The circumstances of students after they became truant are presented in Table 4 . The results reflect the responses of students who had become truant within the period from April 2016 to March 2017. The percentage of students able to return to school and attend classes at least intermittently was greater than $40 \%$ in all departments, and the highest in each department.

Table 5 describes the support activities considered effective for individual truant students. Schools were asked to provide information regarding effective support measures they had offered to students truant from April 2016 to March 2017 but subsequently returned to school. The percentages for "Home visits by classroom teachers and others" and "Parent interviews (counseling) by classroom teachers and others" was high for all departments, and the percentage for "Student interviews (counseling) by classroom teachers and others" was high for the lower and upper secondary departments.

\section{Discussion}

The primary purpose of this study was to produce an outline of the number of truant students, truancy rates, circumstances contributing to truancy, and support systems for truant students. The discussion below is based on a comparison of the findings regarding truant students at elementary, junior high, and high schools.

In the schools for special needs education (ID) surveyed in this study, the percentage of schools with truant students was lower for the elementary department level (27.1\%) and the lower secondary department level (36.4\%), compared to the corresponding percentages for ordinary schools $(40.5 \%$ for elementary school, $85.4 \%$ for lower secondary school, $85.5 \%$ for upper secondary school) (MEXT, 2017b). In ordinary schools, the number of truant students increased by approximately $200 \%$ from sixth grade in elementary school to first grade in lower secondary school, whereas in schools for special needs education (ID), the number of truant students only increased by approximately $50 \%$ from the elementary department to the lower secondary department. The school districts for elementary schools and junior high schools are typically different in Japan, meaning that students are from different elementary schools and study in the same class in junior high schools. As a result, entering junior high school involves significant changes in the school environment, such as in class composition and school structure. By contrast, in schools for special needs education (ID), the students' environment undergoes only small changes as they advance from the elementary to the lower secondary department, which may explain why schools for special needs education (ID) did not observe as dramatic an increase in the number of truant students over this advancement period. Indeed, of the 379 schools that participated in this study, 274 (72.2\%) included both elementary and lower secondary departments (with 36 of the schools also including the upper secondary department); therefore, when the students at these schools advance from the elementary to the lower secondary department, the students likely experience less extensive changes than corresponding students in ordinary schools, principally, it has been posited, because the students at these schools for special needs education (ID) do not have to change schools and, thus, experience less geography-related changes to their schooling environment. In addition, lack of collaboration between elementary and junior high schools has been cited as a factor in the increase in truancy among students who have entered junior high school (MEXT, 2015c). By contrast, in schools for special needs education, teachers and staff members collaborate with each other easily, including sharing information on students and parents, because the staff offices of all the departments are on the same site and in some cases even in the same room or on the same floor. This factor that the teachers can continue to support even after the students go to upper grade is considered a contributing factor for limiting increases in student truancy at the lower secondary department level.

According to MEXT (2018), the truancy rate in ordinary schools $(0.5 \%$ in elementary school, $3.0 \%$ in lower secondary school, $1.5 \%$ in upper second- 
ary school) tends to peak during lower secondary school; whereas, in this study, it increased from the elementary through to the upper secondary department. A part of the reason why the truancy rate in ordinary schools decreased in high school is that a little under $30 \%$ of truant students drop out of high school (MEXT, 2018). In this study, the percentage of truant upper secondary students who dropped out of school was 3.6\% (21 students), and 85.5\% (502 students) of truant upper secondary students had been truant intermittently or continuously for two years or more, with some being truant because they were at the lower secondary department level. These factors appear to contribute to a further increase in student truancy at the upper secondary department level.

In schools for special needs education (ID), the truancy rates for students in the elementary department $(1.2 \%)$ and the upper secondary department (3.8\%) were higher than the corresponding truancy rates for students in ordinary schools. The higher truancy rate, relative to students in ordinary upper secondary schools, among upper department students at schools for special needs education (ID), is considered to be related to the following: Truant students in ordinary upper secondary schools tend to drop out, depressing the truancy rate. An investigation of the causes for the higher truancy rate, relative to students at ordinary elementary schools, for elementary department students at schools for special needs education (ID) is, however, necessary. Notably, although the truancy rate for elementary department students at schools for special needs education (ID) was higher than the corresponding rate at ordinary schools, the percentage, for the elementary department level, of schools for special needs education (ID) with truant students (27.1\%) was much lower than the corresponding percentage for ordinary elementary schools (52.1\%) (MEXT, 2017b).

The literature has suggested that the high proportion of truant students in the upper secondary department is related to the dropout rate; however, because dropping out in the elementary departments is unthinkable, the following factors are conceivable. In the elementary departments, the proportion of schools where truant students were enrolled is $27.1 \%$ (Table 1), which is lower than that of elementary school. Therefore, it is considered that the enrollment rate of truant students per school tends to be high in elementary departments. Additionally, among schools for special needs education (ID), some schools had a higher number of truant students. Those schools might be affected by specific factors, but a discussion of the possible factors would be difficult based on the information collected for this study. The effective supports in elementary departments were "Home visits by classroom teachers and others" and "Parent interviews (counseling) by classroom teachers and others." This finding that effective support requires the cooperation of family suggests that cooperation between parents and schools was crucial. However, in elementary departments, the proportion of respondents who stated "Parents not eager to send students to school" and "Parents do not want their children's teachers" was higher than those of lower and upper secondary departments. These findings suggest that cooperation between teachers and guardians was effective; however, especially in the elementary departments, cooperation was difficult, and we inferred that supporting truant students was sometimes impossible. The survey results for the elementary department level could have been influenced by many factors that have yet to be fully elucidated, such as non-respondent bias and the absence or presence of truancy prevention measures at participating schools, in addition to other factors, such as active accommodation of truant tendencies on the part of some elementary departments and particular biases of the schools that responded to the survey. Careful investigation is therefore needed in this area.

A school committee on truancy was in place at $40.6 \%$ of the responding schools, and 127 schools (33.5\%) collaborated with specialized, external institutions and/or experts, instead of instituting such a committee. The percentage of schools working with off-campus experts is low suggests that support for truant students in schools for special needs education (ID) is typically provided as needed by a limited number of individuals, such as classroom teachers and nursing staff, rather than through coordinated activities performed by dedicated personnel or committees. Ultimately, the best method to provide support to truant students may vary depending on the specific characteristics of students and schools as well as the availability of mechanisms for providing support in a coordinated manner. Therefore, and given that the number of truant students in schools for special needs education has increasing around 1.6 times from 2004 to 2014 (Sonoyama et al., 2017), develop- 
ing methods for coordinated support tailored to individual schools is necessary. A review of the responses regarding which support activities were effective indicates that a combination of support activities was often used, and that establishing a system to effectively coordinate various support activities is critical.

Of the school-related causes of truancy, "Relationships with other students" contributed to the most cases of truancy in all departments, and the "Relationships with teachers" truancy cause contributed to a relatively large number of truancy cases. According to a survey of ordinary schools conducted by MEXT (2017b), 12.9\% of elementary schools, $17.9 \%$ of lower secondary schools, and $14.0 \%$ of upper secondary schools answered "yes" to the question "Did the truant student have relationship problems?" In the present survey, "Relationships with other students" and "Relationships with teachers" are thought to correspond to this data. Therefore, we combined these two factors and used them in a comparison of truancy at ordinary schools and schools for special needs education (ID), wherein we observed that the rate of truancy due to relationship problems for schools for special needs education (ID) is more than twice that for ordinary schools. Human relations problems tend to lead to high-stress reactions (Emura \& Okayasu, 2003; Shimada, Togasaki, Okayasu, \& Sakano, 1996), and the risk of suffering from mental health problems is four times higher for students with ID than for other children (Emerson \& Hatton, 2007). In addition, students who find it challenging to understand the difficulties and rules involved in learning, and/or to play with friends, are more likely to secondarily develop emotional insecurity, apathy, and/or ill feelings toward themselves, all of which increase their risk of truancy (MEXT, 1999). In the survey conducted as part of this study, "Apathy" was the only truancy factor directly related to mental health, and the percentage of truancy cases it contributed to was not particularly high. However, given that the truancy rate had an overall tendency to increase from the elementary to the upper secondary department and that human relations can contribute to mental health problems, we inferred that human relations problems related to students with ID are crucial from the perspective of truancy.

Of the family-related truancy causes, "Parents not eager to send students to school" contributed to a relatively high number of truancy cases at all depart- ment levels, but its level of contribution decreased as students advanced to higher grades; conversely, the impact of the truancy cause, "Parents' difficulty in sending students to school," increased as students advanced to higher grades. When student-related causes are involved, such as "stubborn unwillingness to attend school when they are urged to," it may be more difficult for parents to manage the problem.

The impact of the student-related truancy cause, "Activities for amusement/delinquency," exhibited a different tendency in schools for special needs education (ID) than in ordinary schools. In ordinary schools, this truancy factor contributed to $0.9 \%$ of truancy cases in elementary school, $6.0 \%$ in lower secondary school, and $10.7 \%$ in upper secondary school (MEXT, 2017b). However, in schools for special needs education (ID), its impact was the greatest in the elementary department, contributing to $25.3 \%$ of truancy cases, but then its significance declined sharply in the lower and upper secondary departments, where it only contributed to $4.0 \%$ and $5.3 \%$ of truancy cases, respectively. The reason why the tendency was different from such a usual school was not observed in the description that related to previous research, and further research is necessary to investigate the reason.

The percentage of students who were truant and returned to school during the survey period was roughly $9 \%$ for all department levels. The support activities that contributed to a high percentage of the cases where truants returned to school at all department levels were "Home visits by classroom teachers and others" and "Parent interviews (counseling) by classroom teachers and others," suggesting that an approach by the school to parents was effective for all departments. For the elementary department, "Parents are eager to have their child attend school" contributed to a high percentage of returning truant cases, and for the lower and the upper secondary departments, "Student interviews (counseling) by classroom teachers and others" contributed to a high percentage of such cases. This finding indicates that as students advance to higher grades, the focus of successful support can be shifted from parents to students. Finally, "Support from specialized, external institutions and/or experts" contributed to slightly more than $40 \%$ of the cases where truants returned to school, at all department levels, indicating that this support activity is at least somewhat effective. MEXT 
(2016) states that schools must, in cooperation with welfare and medical institutions, accurately evaluate a truant's home and family situation while taking into account the specific causes of a given student's truancy and other background factors to provide effective support and take effective action, indicating the need for coordinated in-school support mechanisms and for collaboration with specialized, external institutions and/or experts. This study demonstrates that 98 of the schools for special needs education (ID) surveyed (25.9\%) did not collaborate with specialized, external institutions and/or experts; this number must increase to improve the provision of support for truant students. It is not only necessary to promote school collaboration with such institutions and/or experts. The most successful methods of such collaboration must also be identified, and schools must be encouraged to adopt these effective methods by tailoring them to fulfill the needs of specific schools and regions.

The survey conducted as part of this study was, to the best of the authors' knowledge, the first in Japan to investigate truancy in schools for special needs education (ID); additionally, by elucidating such truancy, the survey revealed the different truancy circumstances in the elementary, lower secondary, and upper secondary departments and gathered information on existing in-school support measures for truant students in schools for special needs education (ID), including which measures were considered to be effective. Non-respondent bias should be considered, however, because the data in the study was collected from only $44.8 \%$ of all schools for special needs education (ID) in Japan. In addition, many students with ID have mental health problems, including mental diseases (Einfeld, Ellis, \& Emerson, 2011; van de Wouw, Evenhuis, \& Echteld, 2012), challenging behaviors (Chapman \& Wu, 2012; Vrijmoeth, Monbaliu, Lagast, \& Prinzie, 2012), health conditions (Hilgenkamp, van Wijck, \& Evenhuis, 2012), and/or other developmental disabilities (Edwards, Perlman, \& Reed, 2012). The factors behind the occurrence and continuance of truancy are complicated and must, therefore, be carefully investigated through qualitative research and case studies.

The data in this study were collected from $44.8 \%$ of all schools for special needs education (ID) in Japan and, therefore, must be carefully examined through qualitative research and case studies. Moreover,
Tables 3, 4, and 5 present the data for approximately $63 \%(N=835)$ of all truant students $(N=1,327)$ at participant schools. As aforementioned, those schools were asked to respond about up to five students for the question asking about individual backgrounds, even though they had additional truant students at the time of study. The number of individual cases was limited to reduce the burden on the survey respondents, but the information collected could be more accurate if the participant schools were asked to provide individual backgrounds of all truant students.

\section{Acknowledgment}

We would like to thank the National Association for Principals of Schools for Special Needs Education (Department of Education for Students with Intellectual Disabilities) for their cooperation in conducting the survey. This work was supported by JSPS KAKENHI Grant Number JP16K13594.

\section{References}

Chapman, S. L. C. \& Wu, L. T. (2012) Substance abuse among individuals with intellectual disabilities. Research in Developmental Disabilities, 33, 1147-1156.

Edwards, D. J., Perlman, A., \& Reed, P. (2012) Unsupervised categorization in a sample of children with autism spectrum disorders. Research in Developmental Disabilities, 33, 1264-1269.

Einfeld, S. L., Ellis, L. A., \& Emerson, E. (2011) Comorbidity of intellectual disability and mental disorder in children and adolescents: A systematic review. Journal of Intellectual \& Developmental Disability, 36, 137-143.

Emerson, E. \& Hatton, C. (2007) Mental health of children and adolescents with intellectual disabilities in Britain. The British Journal of Psychiatry, 191, 493-499.

Emura, R. \& Okayasu, T. (2003) Classroom-based social skills education: Junior high school students. Japanese Journal of Educational Psychology, 51, 339-350. (in Japanese)

Hilgenkamp, T. M., van Wijck, R., \& Evenhuis, H. M. (2012) Low physical fitness levels in older adults with ID: Results of the HA-ID study. Research in Developmental Disabilities, 33, 1048-1058.

Ministry of Education, Culture Sports, Science and Technology (1999) Guidance for students with learning disabilities (Report). (in Japanese) http://www.mext.go.jp/a_menu/shotou/tokubetu/ 03110701/005.pdf (Retrieved July 6, 2018)

Ministry of Education, Culture Sports, Science and Technology (2015a) Document on educational counselling at 
school. (in Japanese) http://www.mext.go.jp/b_menu/shingi/ chousa/shotou/120/gijiroku/__icsFiles/afieldfile/2016/ 02/12/1366025_07_1.pdf (Retrieved December 5, 2017)

Ministry of Education, Culture Sports, Science and Technology (2015b) Special needs education 2: Status of special needs education. (in Japanese) http://www.mext.go.jp/a_menu/shotou/ tokubetu/002.htm (Retrieved October 30, 2017)

Ministry of Education, Culture Sports, Science and Technology JAPAN. National Institute for Educational Policy Research (2015c) Leaf over the theory and practice on Seitoshidou: Truth of Gaps Experienced by First Year Students of Junior High School. (in Japanese) http://www.nier.go.jp/shido/leaf/ leaf15.pdf (Retrieved July 5, 2018)

Ministry of Education, Culture Sports, Science and Technology (2016) Support to truant students (notification). (in Japanese) http://www.mext.go.jp/a_menu/shotou/seitoshidou/1375981. htm (Retrieved October 30, 2017)

Ministry of Education, Culture Sports, Science and Technology (2017a) The school basic survey. (in Japanese) http://www. mext.go.jp/b_menu/toukei/chousa01/kihoon/1267995.htm (Retrieved October 30, 2017)

Ministry of Education, Culture Sports, Science and Technology (2017b) Results of 2016 survey on issues regarding student guidance concerning student's behavior problems. (in Japanese) http://www.mext.go.jp/b_menu/houdou/29/10/1397646. htm (Retrieved October 30, 2017)
Ministry of Education, Culture Sports, Science and Technology (2018) 2016 Survey on issues of problem behaviors of students and guidance for truant students. (in Japanese) https:// www.e-stat.go.jp/stat-search/files?page $=1$ \&toukei $=0040030$ $4 \&$ kikan $=00400 \&$ tstat $=000001112655 \&$ cycle $=0 \&$ second $=$ $1 \&$ second2=1 (Retrieved July 7, 2018)

Shimada, H., Togasaki, Y., Okayasu, T., \& Sakano, Y. (1996) The buffering effects of acquired social skills on psychological school stress in elementary school children. Japanese Journal of Behavioral Therapy, 22, 9-19. (in Japanese)

Sonoyama, S., Cho, S., \& Kuramitsu, A. (2017) Preliminary study of non-attendance at schools for special needs education. Japanese Journal of Disability Sciences, 41, 173-182. (in Japanese)

Takagi, R. (1983) Psychology and pathology of stubborn unwillingness to attend school. In K. Uchiyama (Ed.), Stubborn unwillingness to attend school. Kongou Publication, Tokyo, 11-58. (in Japanese)

van de Wouw, E. E., Evenhuis, H. M., \& Echteld, M. A. (2012) Prevalence, associated factors and treatment of sleep problems in adults with intellectual disability: A systematic review. Research in Developmental Disabilities, 33, 1310-1332.

Vrijmoeth, C., Monbaliu, E., Lagast, E., \& Prinzie, P. (2012) Behavioral problems in children with motor and intellectual disabilities: Prevalence and associations with maladaptive personality and marital relationship. Research in Developmental Disabilities, 33, 1027-1038. 